\title{
La empleabilidad de los egresados de comunicación organizacional de la UPAEP. Una respuesta a las necesidades sociales
}

\author{
The employability of the graduates of organizational \\ communication of the UPAEP. A response to social needs
}

Eva María Pérez Castrejón

evamaria.perez@upaep.mx

Proyectos Estratégicos

Universidad Popular Autónoma del Estado de Puebla (UPAEP)

21 Sur, núm.1103, Barrio de Santiago, C.P. 72410, Puebla, Pue., México

Carmen Karina Torrescano de la Peña

carmenkarina.torrescano@upaep.mx

Comunicación y Medios Digitales

Universidad Popular Autónoma del Estado de Puebla (UPAEP)

21 Sur, núm. 1103, Barrio de Santiago, C.P. 72410, Puebla, Pue., México

Editor: Rogelio del Prado Flores

\section{RESUMEN}

En el presente artículo se muestra el nivel de empleabilidad de los egresados de la licenciatura en Comunicación de la línea terminal en comunicación organizacional de cinco generaciones, que abarcan del 2008 al 2016 de la Universidad Popular Autónoma del Estado de Puebla (UPAEP). Se exponen aspectos teóricos, análisis de diferentes bases de datos y de los resultados de las encuestas de los egresados como una forma de entretejer el desarrollo teórico y experiencia universitaria con las necesidades sociales.

Así mismo, este texto propone contrastar los resultados de la empleabilidad de los egresados en comunicación organizacional, sobre las exigencias actuales de la sociedad en que vivimos.

Para esto, se presentarán las aportaciones teóricas de diferentes autores que refuerzan el valor y la trascendencia de la disciplina, así como los antecedentes históricos del plan de estudios y del programa académico.

Palabras clave: comunicación organizacional, especialista, instituciones educativas, egresado y licenciatura en comunicación 


\begin{abstract}
This article shows the level of employability of the graduates of the Communication Degree of the terminal line in organizational communication of five generations, which cover from 2008 to 2016 of the Universidad Popular Autónoma del Estado de Puebla (UPAEP). Theoretical aspects, analysis of different databases and of the results of the surveys of the graduates are exposed as a way to weave the theoretical development and university experience with the social needs.

Likewise, this text proposes to contrast the results of the employability of graduates in organizational communication, on the current demands of the society in which we live.

For this, the theoretical contributions of different authors that reinforce the value and the transcendence of the discipline will be presented, as well as the historical background of the curriculum and the academic program.
\end{abstract}

Keywords: organizational communication, specialist, educational institutions, graduate and communication degree

\title{
INTRODUCCIÓN
}

La presente investigación muestra los cambios que se generan actualmente en la empleabilidad de los egresados de la licenciatura en Comunicación ante el contexto actual. A través de la presentación descriptiva los resultados del nivel de empleabilidad de los egresados de la licenciatura en Comunicación de la línea terminal de comunicación organizacional de la Universidad Popular Autónoma del Estado de Puebla (UPAEP), del año 2008 al 2016.

Los estudiantes de la universidad antes mencionada deciden estudiar el fenómeno de la comunicación desde el inicio de la licenciatura y a partir del séptimo semestre, la incidencia y obtención de conocimientos de la comunicación organizacional es mayor; es entonces, cuando deciden cursar la línea terminal en dicha disciplina, donde obtienen diferentes perspectivas y analizan los contextos que predominan en los entornos organizacionales.

Actualmente, las organizaciones públicas y privadas son absolutamente heterogéneas en su magnitud, en su razón de ser, estructura y objetivos. En México existen cerca de I6,576 organizaciones que al 3 I de diciembre de 20 II contaban con Clave Única de Inscripción al Registro Federal de las Organizaciones de la Sociedad Civil (CLUNI) (Secretaría de Desarrollo Social, 20II). Las organizaciones, de acuerdo con Gustavo León Duarte, se pueden clasificar en privadas, públicas y del tercer sector (León, 2006). 
Los universitarios del programa académico en Comunicación egresan con herramientas que les posibilitan analizar las estrategias de comunicación implementadas en las organizaciones públicas o privadas, y así decidir las acciones de comunicación organizacional que puedan incidir favorablemente en los públicos internos y externos.

El trabajo se presenta metodológicamente desde una perspectiva cuantitativa, a través de herramientas como la encuesta a egresados de la línea disciplinar y el análisis de contenido de los resultados de bases de datos de repositorios de la UPAEP.

A través de los resultados obtenidos de la investigación se llevaron a cabo los análisis oportunos de los resultados del nivel de empleabilidad de los egresados de la licenciatura en Comunicación de la línea terminal de comunicación organizacional de la UPAEP. Todo esto, para analizar los entornos actuales donde los egresados tengan entrada a laborar.

\section{HALLAZGOS}

Una vez que se analizaron las bases de datos de la UPAEP y encuestaron a los 49 egresados de la línea terminal en comunicación organizacional, se presentan los resultados del nivel de empleabilidad de los egresados, de quienes se obtuvo información acerca de las organizaciones donde trabajan, cargo, su ubicación geográfica, el rol que desempeñan y sus actividades principales. Dichos resultados posibilitaron percibir un nivel de experiencia y las competencias profesionales que les han permitido desenvolverse de manera competente en el ámbito laboral.

El especialista en comunicación organizacional egresado de la UPAEP es un analista que se desarrolla en la práctica organizacional, diseña herramientas acordes con las necesidades de cada organización, aplica diagnósticos, visualiza los cambios o ajustes en áreas de oportunidad, además de preocuparse por reforzar la identidad corporativa y fortalecer el comportamiento organizacional a partir de una filosofía organizacional ya establecida.

\section{MARCO TEÓRICO}

Esta investigación contribuye con las instituciones de educación superior, que ofrecen la licenciatura en Comunicación con énfasis en la disciplina de la comunicación organizacional, a detectar e interesarse en saber si sus egresados se están empleando en organizaciones donde su rol esté íntimamente relacionado con la disciplina ya mencionada; de lo contrario, quizás habría que realizar una propuesta de contenidos a través de sus planes de estudio, ya 
que las organizaciones han de responder a sus clientes con decisiones colectivas previamente establecidas en sus objetivos planteados como una respuesta social.

\section{LOS PROGRAMAS ACADÉMICOS EN COMUNICACIÓN Y SU IMPORTANCIA} EN LA FORMACIÓN DE ESPECIALISTAS EN LA DISCIPLINA

Los programas académicos en Comunicación con línea especializada en comunicación organizacional necesitan la intervención de estrategias que respondan a los desafíos que plantea la misión de la comunicación moderna. Si dichas estrategias se tornan creativas y resolutivas, esto puede ser prueba que el aprendizaje fue significativo y por lo tanto es pertinente en las organizaciones. De acuerdo con la teoría de los campos de Bourdieu, la noción de babitus del profesional (2003, p. II3), permite analizar la estructura que rige el campo de la licenciatura en Comunicación.

De acuerdo con un estudio realizado en el 2008 por María Antonieta Rebeil, existen más de mil programas de comunicación o sus similares que ofertan las universidades en México; en el estado de Puebla hay 42 programas académicos que ofertan dicho programa y en Puebla capital 32 programas (Superior 2019). A diferencia de la Asociación Nacional de Universidades e Instituciones de Educación Superior (ANUIES) que reporta la existencia de 445 planes de estudios ofertados en México referente a Comunicación, Ciencias de la Comunicación, Ciencias y Técnicas de Comunicación, Comunicación y Medios Digitales, Periodismo, Periodismo Digital, entre otras (ANUIES, 2019).

La comunicación organizacional en México está irrumpiendo de manera integral en las organizaciones públicas y privadas como parte de los procesos laborales con directrices previamente determinadas, esto con la intención de lograr una mayor competencia. Esta comunicación ha generadouna vital importancia para las buenas relaciones de los colaboradores en su entorno interno y externo. También de establecer y conseguir las metas y los objetivos que deben alcanzar. Además, como menciona Raúl Fuentes Navarro (2012), al ser una disciplina relativamente nueva y con mucho que aportar al país, las instancias educativas juegan un rol fundamental, en una reflexión epistemológica y socio-histórica sobre su propio quehacer, ya que el campo académico de la comunicación aporta en la construcción de dicha disciplina.

Los programas académicos de comunicación organizacional en el país están evolucionando cada día, ya que crece el número de instituciones que se suman para ver a esta como una posibilidad de crecimiento de los especialistas de la comunicación, este tipo de comunicación posibilita la práctica dentro y fuera de las organizaciones. El buen funcionamiento de las redes internas debe ser paralelo a las que se tiene con el cliente externo (Guzmán, 20I2). 
El estudio de la comunicación organizacional en México desde los diferentes programas académicos se ha enfocado a la diversidad de organizaciones desde las más grandes, las medianas y las pequeñas, donde la primicia y las acciones innovadoras se han convertido en un tema de gran utilidad en el espacio académico, a través del cual los estudiantes pueden crear, transferir e integrar conocimientos al servicio común donde se asegure el diálogo abierto y argumentado entre los miembros de la organización y los diferentes sistemas. Esto requiere de un lenguaje común que permita un espacio de encuentro transparente, abierto y comprometido al ejercer sus funciones en la evolución de la organización como parte de un todo (Queris, Cabrera, García, y Alfonso, 2012).

La ciudad de Puebla de Zaragoza es una de las zonas urbanas más grandes del país, localizada en el altiplano central de México; en los últimos años se ha convertido en el espacio universitario por excelencia que alberga alrededor de 364 universidades, de las cuales 32 ofrecen la licenciatura en Comunicación o sus similares (Pública, 2019), donde el objetivo es dar respuesta a la demanda social en materia de comunicación.

Los programas enfocados a la comunicación organizacional son un elemento que media entre los distintos sistemas organizacionales buscando permanentemente la mejora de las organizaciones, no con la intención de juzgar sino favorecer a los clientes internos y externos, ya que las organizaciones se someten a luchas, como la adecuada tarea del manejo del conocimiento, la información y las interrelaciones (Peiró y Bresó, 20I2).

Para continuar, las instituciones de educación superior que ofrecen programas en comunicación y sus especialistas han tratado de muchas maneras introducir contenidos educativos o culturales a través de diferentes canales de comunicación, con la intención de comunicar de una misma forma contenidos y propósitos con impactos diversos. Por otro lado, queda claro que lo que sí es fundamental en el fenómeno de la comunicación es el saber hacer entre los individuos participantes (Fuentes, 20I2).

\section{EGRESADOS DE LOS PROGRAMAS DE COMUNICACIÓN}

De los egresados universitarios especialistas en comunicación, una menor proporción son especialistas en comunicación organizacional. De acuerdo con el presidente del Consejo para la Enseñanza y la Investigación de las Ciencias de la Comunicación (CONEICC), solo son cuatro programas formalmente establecidos que se dedican a la formación de especialistas en la disciplina y cada uno con diferentes denominaciones, como relaciones públicas, comunicación estratégica, comunicación organizacional, etcétera. Los egresados son quie- 
nes tienen el desafío de posicionar a los especialistas de la mencionada disciplina después de obtener, en una instancia universitaria, conocimientos teóricos que llevan a la práctica como un concepto no reduccionista, ajeno a la realidad, como base de la formación en un esfuerzo ante las necesidades en materia de comunicación de las organizaciones. Tratando de encontrar las mejores maneras productivas de trabajar desde la comunicación organizacional, es decir, buscar procedimientos para generar preguntas pertinentes y respuestas con argumentos socialmente válidos (Pérez, 20ı8).

El término de egresado se puede asociar al espacio estudiantil, se hace referencia a concluir los estudios superiores universitarios, donde se obtuvo un título con pertinencia social y el estudiante sale del centro educativo que lo formó. El egresado tiene una ocupación, conocimientos y habilidades, aporta socialmente a través de sus conocimientos, ofrece un servicio a la sociedad, cumple responsabilidades, se apega a una ética profesional, es experto en un área específica, entre otros (Díaz, I999).

El concepto de egresado especialista en comunicación organizacional también es asociado con las universidades en la formación de profesionales y tiene como objetivo fundar en el educando el rol de la disciplina como la actividad que se crea para reconocer las necesidades de comunicación en las organizaciones, mismas que van más allá de ser una función meramente administrativa. La comunicación organizacional implica un proceso de investigación que posibilita la comprensión clara de aportaciones estratégicas como una respuesta a la competitividad de las organizaciones frente a una realidad que es producto de la globalización, se pide conocimiento extenso de tácticas y estrategias viables para la realidad de cada organización (Pérez, 2018).

La trayectoria del especialista en comunicación se ha caracterizado por no tener en su mayoría incidencia en espacios de comunicación, situación que no es positiva porque es para lo que fueron formados. La tendencia nacional reportada por el Observatorio Laboral Mexicano (2019) informa que de los programas en comunicación y periodismo están ocupados 20I,87 I miles de personas, dichas cifras son reportadas hasta el tercer trimestre de 2018 en la Encuesta Nacional de Ocupación y Empleo, STPS-Inegi, de los cuales 50.3\% son hombres y $49.7 \%$ son mujeres, quienes tienen un salario promedio de \$II,520 (once mil quinientos veinte pesos). Estos resultados llegan a provocar que los egresados de la licenciatura en Comunicación al no encontrar un espacio laboral acorde a su profesión busquen áreas laborales no congruentes con su formación académica, por lo que continuamente se emplean en espacios no relacionados con su profesión (Sánchez, 20 I4). De acuerdo a la base de datos de la ANUIES del ciclo escolar 20I7-20I8, han egresado un total de II,33i alumnos.

La licenciatura en Ciencias de la Comunicación en la UPAEP inició en I983 para formar profesionales en los medios de comunicación, a partir de entonces, el programa académico 
ha tenido seis rediseños curriculares en los que ha evolucionado tratando de incidir correctamente ante las necesidades y las demandas sociales. En el 200I, esta casa de estudios, preocupada por dar una respuesta ante las necesidades de comunicación en las organizaciones poblanas, replantea su programa demostrando la necesidad que se tiene de formar especialistas que creen sistemas de comunicación adecuados y pertinentes al país, con un enfoque a resolver problemas específicos (Puebla, 2000). El documento del rediseño curricular plantea que los estudiantes deben contar con una formación integral, cuidando los conocimientos teóricos, la formación humanística, el dominio tecnológico y la preparación del comunicador como profesional que resuelve problemas específicos en tres distintos ámbitos de acción: el corporativo u organizacional, el político y la comunicación para el desarrollo.

Por lo que, del 2012 al 20I6, han egresado cinco generaciones que hacen un total de 49 especialistas en comunicación organizacional, ya que México y Puebla, en particular, requieren de profesionales que sepan resolver problemas de comunicación de forma integral. Las sociedades necesitan comunicarse de la forma más eficiente, tanto con sus públicos internos como externos. La comunicación adecuada permitirá transformar a las comunidades en organizaciones más productivas, transparentes y contribuirá a que los egresados puedan tener mejores oportunidades de vida.

Ante este panorama, surge el interés por indagar cuál es la empleabilidad de algunos egresados de comunicación organizacional de la UPAEP, para insertarse al mercado laboral, teniendo en cuenta la competencia que se presenta con egresados de otras instituciones y el contexto laboral actual en la ciudad de Puebla y en nuestro país, México.

\section{LA COMUNICACIÓN ORGANIZACIONAL EN MÉXICO}

La comunicación en las organizaciones nace ante la necesidad de las organizaciones públicas y privadas de mejorar los escenarios del desarrollo personal y profesional de los colaboradores, esto con el fin último de coadyuvar al logro de los objetivos de dicha organización. La comunicación organizacional era concebida como la responsable de "la generación de procesos de intercambio que unifican y consensan criterios de productividad y provocan la cooperación y la colaboración colectiva organizada hacia las metas de la organización” (Rebeil y Ruiz Sandoval, I998, p. 15). Posteriormente, se entiende como un proceso estratégico que tenía como objetivo la mejora continua, considerando siempre las necesidades de su audiencia corporativa y social (Rebeil, 2006). Y más adelante también se hizo la reflexión sobre la comunicación integral para las organizaciones socialmente responsables, en cuyo énfasis se encuentra en lograr el compromiso ético de "llevar a vivir una auténtica cultura 
de comunicación y de RS, con el fin de potenciar a la organización en todos sus niveles” (Rebeil y Sánchez, 20I2, p. 47).

Es importante mencionar que la comunicación organizacional ha tenido distintos nombres, como comunicación institucional, comunicación corporativa, comunicación interna, relaciones públicas, entre otros. Se llama comunicación integral para las organizaciones "en tanto incluye de manera integrada las distintas dimensiones de la comunicación que establece una organización con sus grupos de interés” (Rebeil y Moreno, 20I4). La comunicación organizacional incluye tres dimensiones: comunicación institucional (imagen), comunicación interna (gestión) y comunicación mercadológica (ventas). En este sentido, lograr la identificación de los empleados, estrategias y líderes con la visión, los valores y la cultura organizacional es una de las responsabilidades que tiene la comunicación corporativa (Rebeil, 2006). Por otra parte, como resultado de la globalización y el incremento en el uso de la tecnología, la comunicación entre personas y organizaciones físicamente dejó de ser un problema y se convirtió en una fortaleza que hizo posible que las organizaciones pudieran ser parte de proyectos globales a favor de cambios en el ámbito social, económico y político de México. En este contexto, el comunicador para ser eficaz debe cumplir con ciertas funciones: conocer y apoyar la estrategia de negocio, identificar oportunidades, entender necesidades de cambio, anticiparse a problemas, lograr un entendimiento de los mensajes y contar con una tónica emocional para propiciar la motivación y el silencio, según sea necesario (Rebeil, 2006).

\section{LA COMUNICACIÓN ORGANIZACIONAL DESDE EL CAMPO ACADÉMICO}

Desde hace 56 años existen en México programas académicos enfocados al estudio de la Comunicación (Chong, 20I6, en Fuentes, 20II). Sin embargo, en nuestro país, la enseñanza de la comunicación organizacional se da hasta finales de la década de los años sesenta cuando fue integrado a la estructura curricular de algunas universidades de acuerdo con lo que señala Gustavo León (2006, p. 288).

A través de los años, los contenidos de los programas con énfasis en Comunicación Organizacional se han ido desarrollando con la intención de brindar a los estudiantes las habilidades, herramientas y valores que requiere el profesional de la comunicación organizacional. La creación de programas interinstitucionales, como fue el Consejo Nacional para la Enseñanza y la Investigación de las Ciencias de la Comunicación (CONEICC) en I976 y la Asociación Mexicana de investigadores de Comunicación (AMIC) en I979, en 
ésta área se ha conformado el Grupo de Investigación y Comunicación Integral para las Organizaciones.

\section{MARCO CONTEXTUAL}

\section{Antecedentes históricos del plan y programa de estudios propuesto en el ámbito local, nacional e internacional}

La Universidad Popular Autónoma del Estado de Puebla, fundada en I973, ofertó por primera vez la licenciatura en Ciencias de la Comunicación en I983 para satisfacer las demandas de profesionales en el área, teniendo como resultado que muchos egresados hoy formen parte de la élite periodística y de medios de comunicación poblana.

El programa de Ciencias de la Comunicación ha tenido seis revisiones curriculares, la más reciente, la de 20I3, plan 02. En 2008 se cambió el nombre a licenciatura en Comunicación constituyéndose el plan or. El plan de estudios buscaba responder a una necesidad social específica que era la formación profesional de especialistas en comunicación con un enfoque de intervención en la región sureste del país. Sin embargo, persiste la necesidad de desarrollar un fundamento epistemológico explícito, lo cual requiere de trabajo colegiado constante para definir los conceptos básicos del plan de estudios y de la profesión. Cambiar el paradigma de la formación de comunicólogos obliga a contar con un plan de estudios de mayor proyección, una planta docente apegada al perfil requerido y proyectos de vinculación social que permitan a los estudiantes intervenir en las necesidades del mundo global.

El cambio social, transformado además por el acelerado avance de las tecnologías que día a día se vive en contextos locales, regionales, nacionales e internacionales, invita a repensar las tendencias vigentes y futuras de los contenidos del programa de estudios sobre Comunicación en la UPAEP. Haciendo una revisión histórica, desde I983, la Facultad de Comunicación de la UPAEP ha mostrado evolución en sus propuestas académicas, siempre con el objetivo de formar profesionales de la comunicación.

Partiendo de las Ciencias Políticas, el primer programa de estudios en la UPAEP mostró una fuerte formación periodística de los estudiantes. En I995 se buscó ampliar la formación mediante cursos complementarios sobre comunicación organizacional, comunicación educativa, mercadotecnia, entre otras. A partir de las necesidades planteadas por el mercado, en el 200 I se replanteó el programa de estudios separando la licenciatura en Periodismo y la licenciatura en Comunicación (Escuela de Comunicación, 2008). Finalmente, la licenciatura en Periodismo tiene que cerrarse por la baja matrícula. En el plan or (2008) se incorporan 
líneas terminales en la currícula: Comunicación Política, Comunicación Organizacional y Comunicación para el Desarrollo, con la finalidad de ofrecer nuevos campos laborales a los egresados y se reduce el énfasis en la producción de productos audiovisuales, debido a la saturación en el campo de los medios de comunicación. En el plan o2 se mantienen las líneas terminales, se incorporan nuevamente materias de Periodismo, se fortalece un poco más la línea de producción audiovisual, pero se le da más énfasis a la formación de egresados como “interventores de la comunicación”. Por ello, en este último plan de estudios, la línea de investigación es fuerte.

El mercado laboral requiere de profesionales con conocimientos en los campos de teorías y nuevas tecnologías aplicadas a la comunicación, capaces de desarrollar planes estratégicos en contextos sociales diversos con una actitud proactiva y un amplio sentido de la responsabilidad. De ahí la necesidad de un nuevo rediseño que haga mayor énfasis en el manejo del software y las plataformas digitales, para la realización de productos multimedia digitales, sin dejar de lado su formación en las teorías de la comunicación, en el conocimiento del contexto social, en la reflexión y el análisis crítico, a fin de generar contenidos de calidad.

La presente investigación se desarrolla en la ciudad de Puebla de Zaragoza, a través de egresados de la Licenciatura en Comunicación de la UPAEP, de las generaciones que abarcan del 2008 al 20I6, cinco generaciones en total. Los estudiantes cursan nueve semestres, por lo que concluyen los estudios universitarios en un promedio de cuatro años y medio. Son 2II alumnos de nuevo ingreso en estas generaciones, de los cuales 73 son hombres y I3 8 mujeres. A la fecha están titulados 87 alumnos y sin titular 17 , esto indica que desertaron I07 estudiantes de estas generaciones. Los alumnos de los que se mostrará el nivel de empleabilidad son los que seleccionaron la línea terminal en comunicación organizacional y hacen un total de 49 personas.

\section{METODOLOGÍA}

La metodología de investigación que se utiliza es cuantitativa, ya que se emplearon técnicas como: análisis y reportes de bases de datos. Este tipo de metodología es resultado de un estudio cuantitativo ya que muestra el control sobre el fenómeno a investigar y posibilita un conteo de los resultados. En esta investigación se diseñó un instrumento para la recolección de datos y se aplicó la encuesta a los egresados que aceptaron participar.

El marco muestral son los egresados de la licenciatura en Comunicación de la línea terminal en comunicación organizacional de la UPAEP, que del 2012 al 2016 suman 49 alumnos, mismos que sirvieron en su totalidad como muestra. 
Se realizó un análisis de las bases de datos de los repositorios de la misma casa de estudios y se aplicó una breve encuesta a los egresados a través de la cual se les cuestionó acerca de: género, edad, año de egreso, lugar de trabajo, el giro de la organización, el rol que desempeñan, tres actividades principales que realicen y la ciudad y municipio en el que radican.

La investigación cuantitativa obtiene resultados lógicos, numéricos y estadísticos, como procedimiento que establece las magnitudes numéricas. Se apoya del canon que menciona: las partes representan el todo; estudiando una muestra nos da la idea de cómo está la población en su conjunto (Hueso y Cascant, 2012).

Para realizar la investigación se consideró el Modelo de Investigación que se presenta en la Figura I.

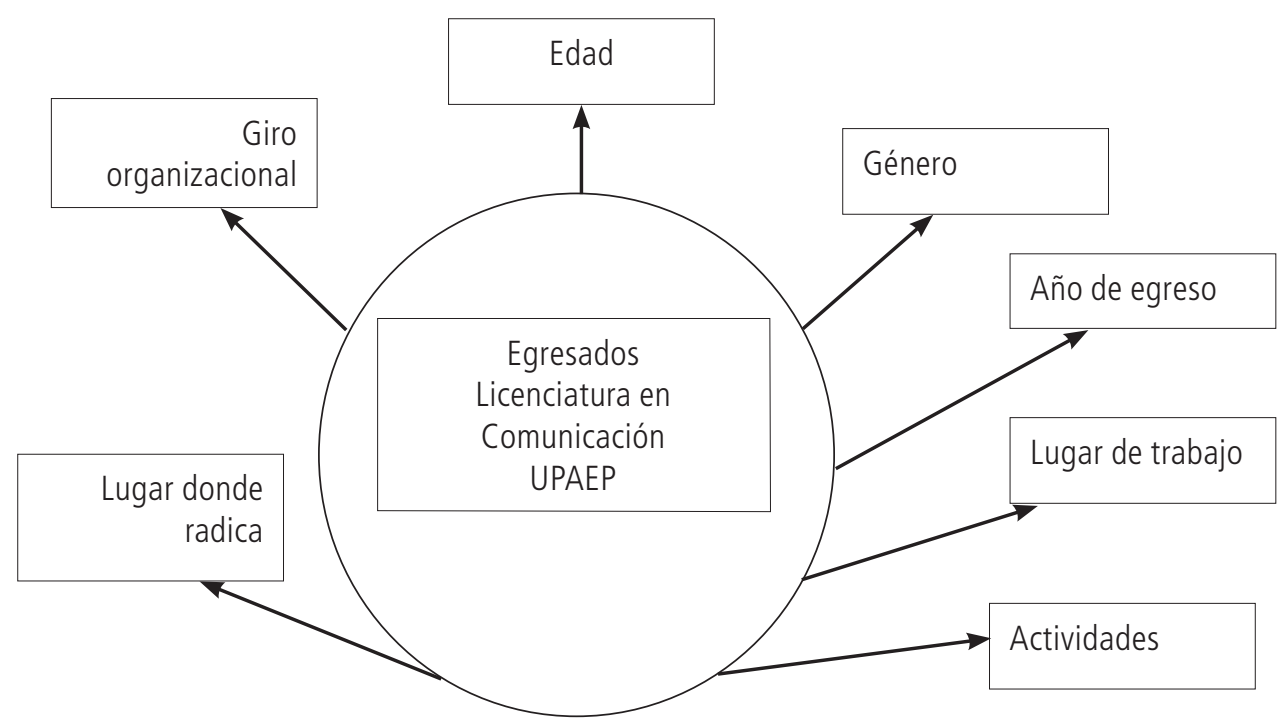

FIGURA 1. MODELO DE INVESTIGACIÓN

FUENTE: ELABORACIÓN PROPIA, 2019.

\section{ANÁLISIS DE DATOS}

La Gráfica i muestra las estadísticas de las últimas cinco generaciones (2008-2016). De un total de 2 II alumnos de ingreso, egresaron únicamente I04, de los cuales 87 son los egresados titulados y 17 sin titular. 
GRÁFICA 1. ESTADÍSTICAS ÚLTIMAS CINCO GENERACIONES

\begin{tabular}{|c|} 
Universidad Popular AutónOMA DEL ESTADO DE PUEBLA A.C. \\
\hline Colicitada por el Decanato de Ciencias Sociales \\
\hline Comunicación \\
\hline Duración de 4.5 años/9 semestres \\
\hline
\end{tabular}

Estadísticas últimas 5 generaciones

\begin{tabular}{|r|c|c|c|c|c|c|c|}
\hline & $2008-2012$ & $2009-2013$ & $2010-2014$ & $2011-2015$ & $2012-2016$ & TOTAL & $\%$ \\
\hline N. ${ }^{\circ}$ INGRESOS & 53 & 46 & 38 & 39 & 35 & 211 & \\
\hline Mujeres & 34 & 34 & 25 & 28 & 17 & 138 & 65 \\
\hline Hombres & 19 & 12 & 13 & 11 & 18 & 73 & 35 \\
\hline Egresados sin titular & 2 & 0 & 3 & 7 & 5 & 17 & 16 \\
\hline Egresados titulados & 31 & 24 & 15 & 12 & 5 & 87 & 84 \\
\hline Total egresados & 33 & 24 & 18 & 19 & 10 & 104 & \\
\hline
\end{tabular}

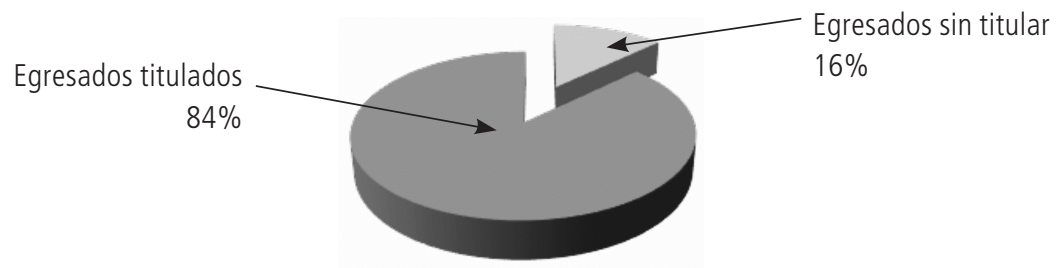

Análisis longitudinal (análisis de la información a través del tiempo en forma acumulada)

\begin{tabular}{|c|c|c|c|c|c|c|c|c|}
\hline $\begin{array}{l}\text { AÑO DE INGRESO } \\
\text { ** (GENERACIÓN) }\end{array}$ & $\begin{array}{l}\text { EGRESADOS } \\
\text { EN TIEMPO }\end{array}$ & $\begin{array}{c}\text { EGRESADOS } \\
\text { 1ER. AÑo }\end{array}$ & $\begin{array}{l}\text { EGRESADOS } \\
\text { 2DO. AÑO }\end{array}$ & $\begin{array}{c}\text { EGRESADOS } \\
\text { 3ER. AÑO }\end{array}$ & $\begin{array}{l}\text { TITULADOS } \\
\text { 1ER. AÑO }\end{array}$ & $\begin{array}{l}\text { TITULADOS } \\
\text { 2DO. AÑO }\end{array}$ & \begin{tabular}{|l} 
TITULADOS \\
3ER. AÑo
\end{tabular} & $\begin{array}{l}\text { TITULADOS } \\
\text { 4TO. AÑO }\end{array}$ \\
\hline $2008-2012$ & 12 & 28 & 32 & 33 & 17 & 22 & 29 & 31 \\
\hline $2009-2013$ & 12 & 20 & 22 & 24 & 12 & 16 & 22 & 24 \\
\hline $2010-2014$ & 7 & 16 & 18 & 18 & 9 & 15 & 15 & NA \\
\hline 2011-2015 & 7 & 19 & 19 & NA & 10 & 12 & NA & NA \\
\hline $2012-2016$ & 10 & 16 & NA & NA & 5 & NA & NA & NA \\
\hline
\end{tabular}

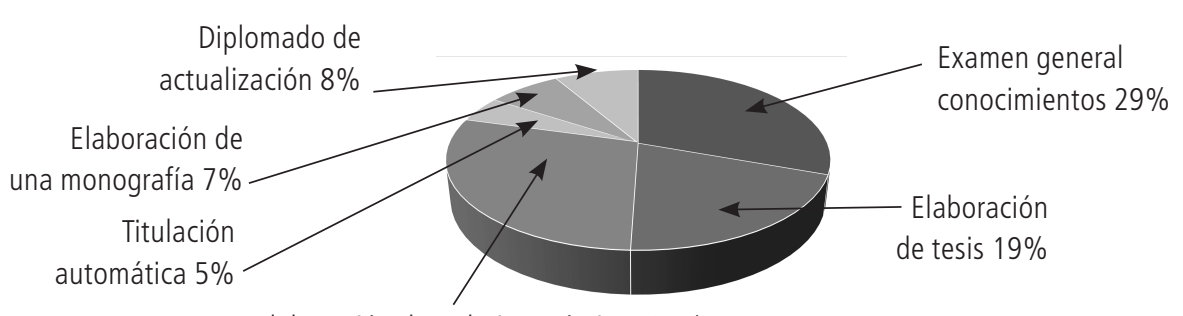

Elaboración de trabajos prácticos $32 \%$

\section{Dirección de Efectividad Institucional}

Elaboró: Mtra. María Elena Lara Rodríguez

Resumen estadístico Egresados

FUENTE: ELABORACIÓN DEL AUTOR CON DATOS DE LA DIRECCIÓN DE EFECTIVIDAD INSTITUCIONAL DE LA UPAEP. 
En la Gráfica 2 se muestra el total de alumnos por generación del 2008 al 2012. De un total de 33 alumnos el $58 \%$ de alumnos egresaron de la línea terminal de comunicación organizacional, mientras que el $30 \%$ de comunicación para el desarrollo y un I2 \% de política.

GRÁFICA 2. TOTAL DE ALUMNOS POR GENERACIÓN 2008-2012

PROGRAMA ACADÉMICO: COMUNICACIÓN

GENERACIÓN: OTOÑO 2008-OTOÑO 2012

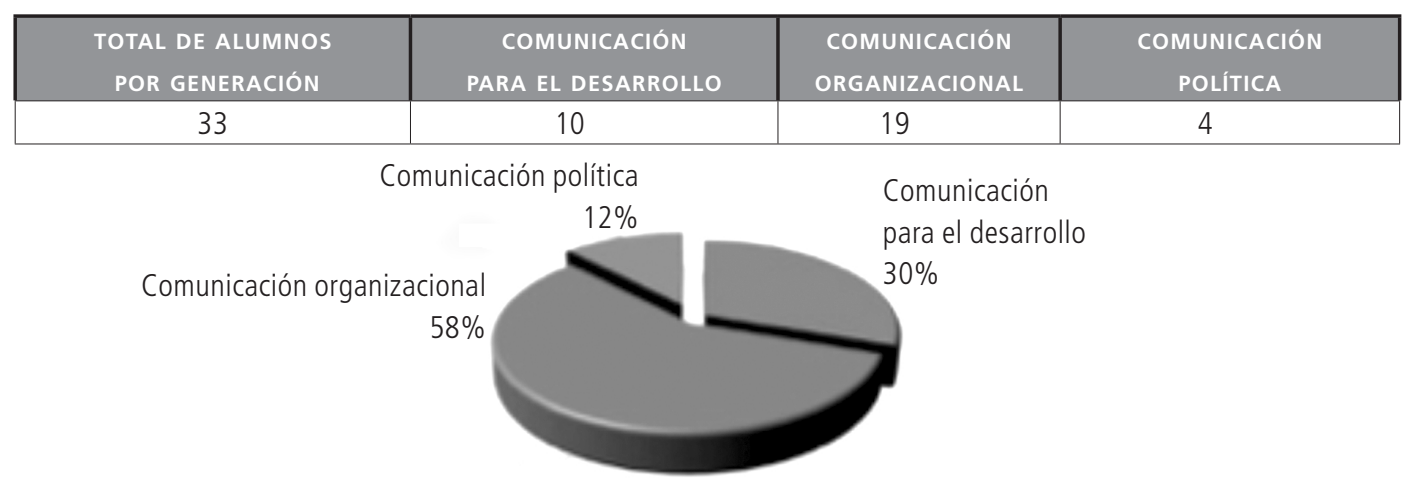

FUENTE: ELABORACIÓN DEL AUTOR CON DATOS DE LA DIRECCIÓN DE EFECTIVIDAD INSTITUCIONAL DE LA UPAEP.

En la Gráfica 3 se muestra el total de alumnos por generación del 2009 al 2013. De un total de 24 alumnos el $46 \%$ egresaron de la línea terminal de comunicación para el desarrollo, el $29 \%$ de comunicación para el organizacional y un I2\% de política.

GRÁFICA 3. TOTAL DE ALUMNOS POR GENERACIÓN 2009-2013

PROGRAMA ACADÉMICO: COMUNICACIÓN GENERACIÓN: OTOÑO 2009-OTOÑO 2013

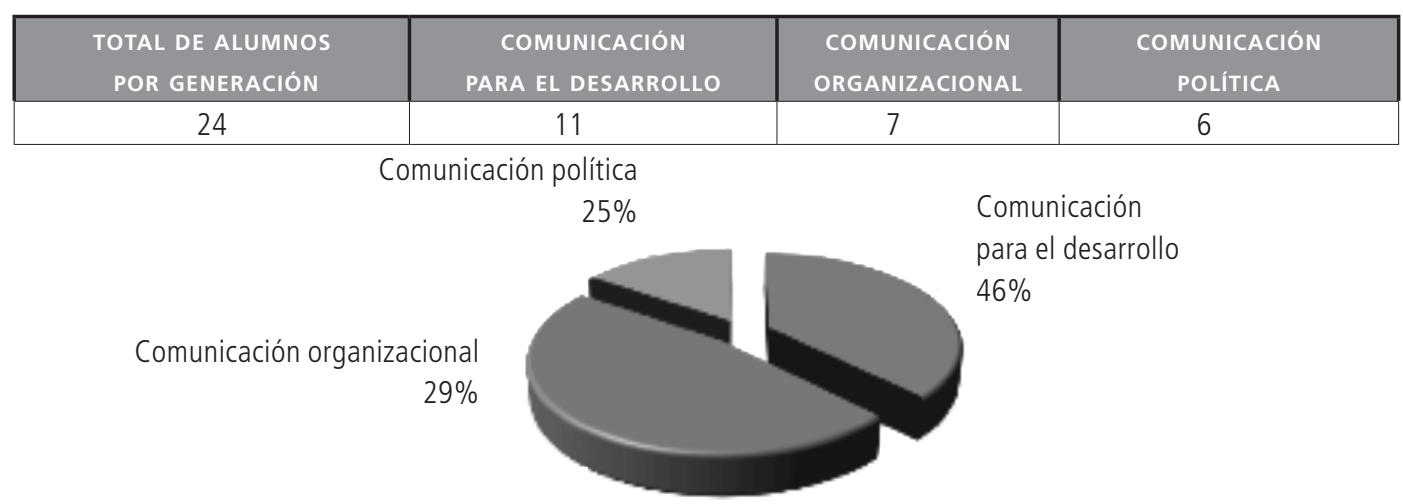

FUENTE: ELABORACIÓN DEL AUTOR CON DATOS DE LA DIRECCIÓN DE EFECTIVIDAD INSTITUCIONAL DE LA UPAEP. 
En la Gráfica 4 se presenta el total de alumnos por generación del 2010 al 20I4. De un total de i 8 alumnos, el $50 \%$ egresaron de la línea terminal de comunicación organizacional, el $33 \%$ de comunicación para el desarrollo y un $17 \%$ de política.

GRÁFICA 4. TOTAL DE ALUMNOS POR GENERACIÓN 2010-2014

PROGRAMA ACADÉMICO: COMUNICACIÓN

GENERACIÓN: OTOÑO 2010-OTOÑO 2014

\begin{tabular}{|c|c|c|c|}
\hline $\begin{array}{l}\text { TOTAL DE ALUMNOS } \\
\text { POR GENERACIÓN }\end{array}$ & $\begin{array}{c}\text { COMUNICACIÓN } \\
\text { PARA EL DESARROLLO }\end{array}$ & $\begin{array}{l}\text { COMUNICACIÓN } \\
\text { ORGANIZACIONAL }\end{array}$ & COMUNICACIÓN POLÍTICA \\
\hline 18 & 6 & 9 & 3 \\
\hline Comunicación & $\begin{array}{r}\text { municación política } \\
17 \%\end{array}$ & $\begin{array}{l}\text { Comunic } \\
\text { para el d } \\
33 \%\end{array}$ & án \\
\hline
\end{tabular}

FUENTE: ELABORACIÓN DEL AUTOR CON DATOS DE LA DIRECCIÓN DE EFECTIVIDAD INSTITUCIONAL DE LA UPAEP.

En la Gráfica 5 se puede observar el total de alumnos por generación del 20Ir al 2015. De un total de ig alumnos, el $53 \%$ egresaron de la línea terminal de comunicación para el desarrollo, el $37 \%$ de comunicación organizacional y un Io\% de política.

GRÁFICA 5. TOTAL DE ALUMNOS POR GENERACIÓN 2011-2015

PROGRAMA ACADÉMICO: COMUNICACIÓN

GENERACIÓN: OTOÑO 2011-OTOÑO 2015

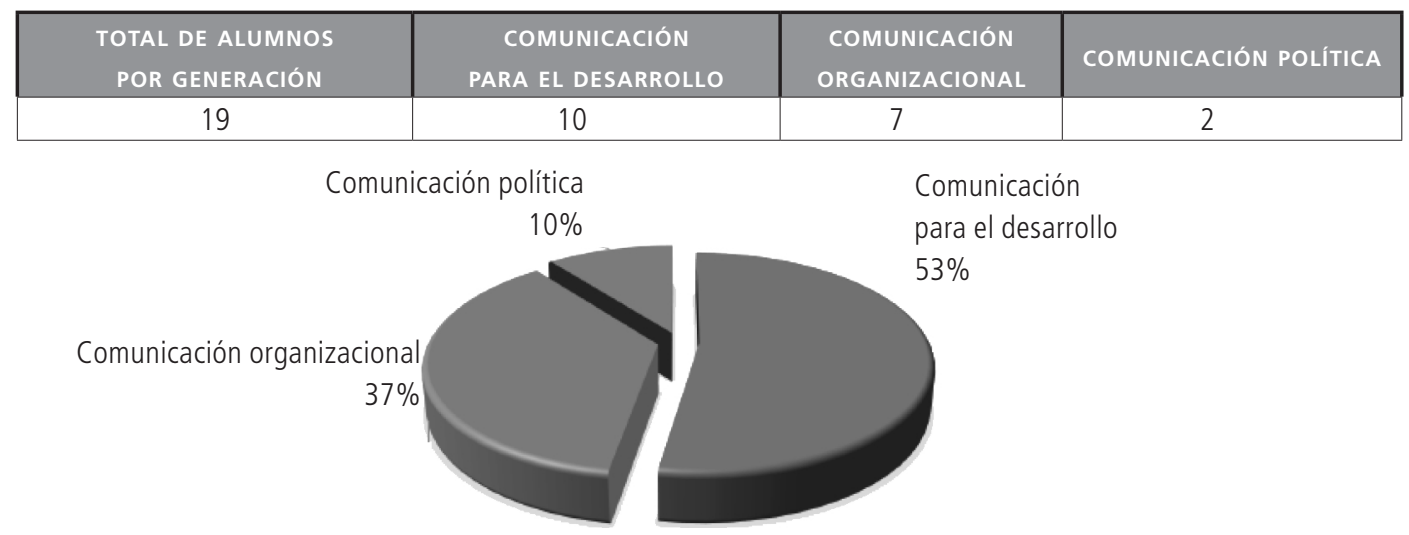

FUENTE: ELABORACIÓN DEL AUTOR CON DATOS DE LA DIRECCIÓN DE EFECTIVIDAD INSTITUCIONAL DE LA UPAEP. 
En la Gráfica 6 se muestra el total de alumnos de las cinco generaciones que nos permite visualizar el porcentaje global con un $47 \%$ de alumnos egresados de la línea terminal de comunicación organizacional, $38 \%$ de comunicación para el desarrollo y $15 \%$ de comunicación política.

GRÁFICA 6. TOTAL DE ALUMNOS DE LAS CINCO GENERACIONES

PROGRAMA ACADÉMICO: COMUNICACIÓN GENERACIÓN: OTOÑO 2012-OTOÑO 2016

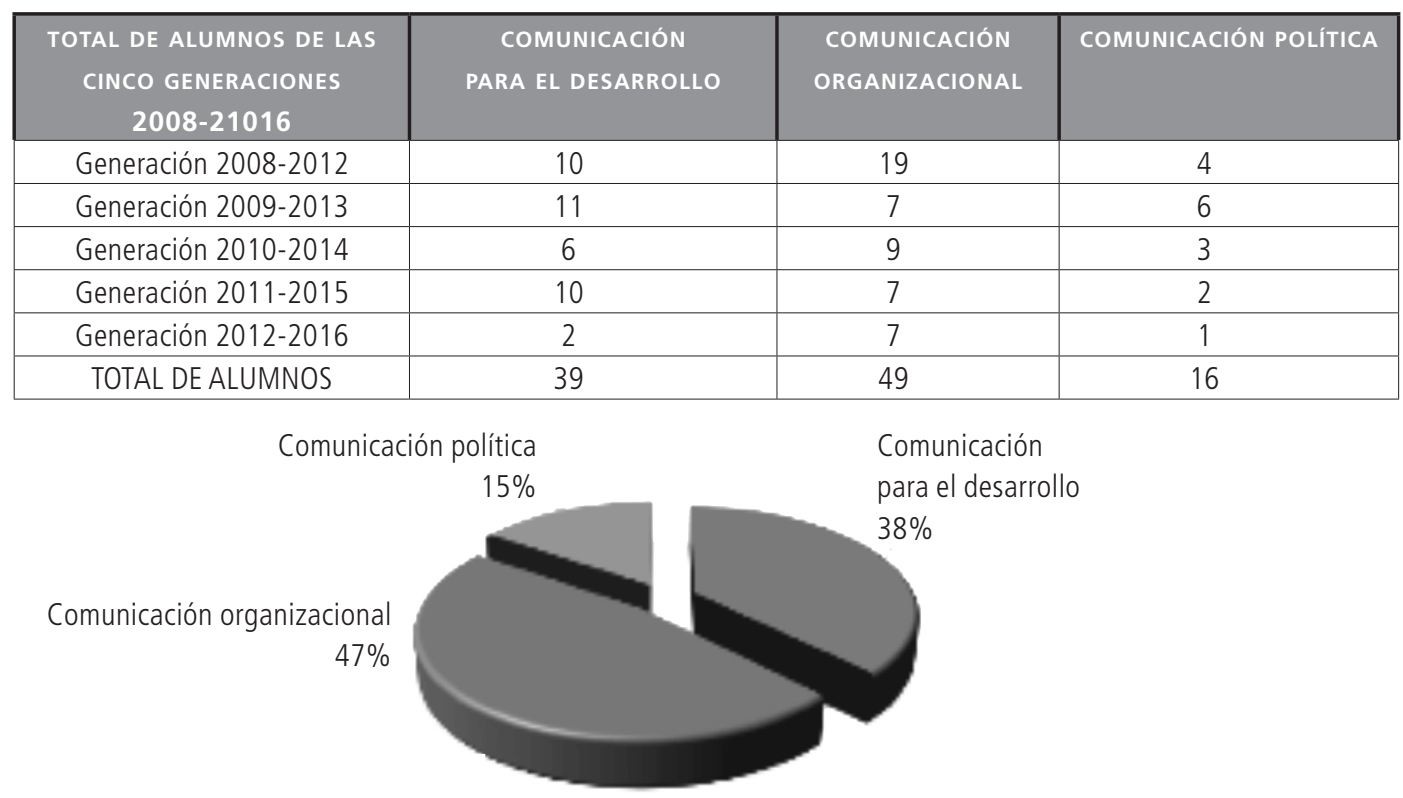

FUENTE: ELABORACIÓN DEL AUTOR CON DATOS DE LA DIRECCIÓN DE EFECTIVIDAD INSTITUCIONAL DE LA UPAEP.

\section{RESULTADOS}

La población estuvo conformada por un total de 49 alumnos egresados de la línea terminal de comunicación organizacional, los cuales representan cinco generaciones (201220r6). Se enviaron 45 cuestionarios electrónicos, ya que fueron los egresados que se pudieron localizar. De los cuales 29 fueron respondidos por $79.3 \%$ de mujeres y $21.4 \%$ de hombres. La edad más representativa (20.7\%) tiene 29 años de edad, seguidos de $17.2 \%$ con 28 años. El 39.2\% radica en Puebla y el año de egreso más representativo que contestó la encuesta fue el 2014. 
El giro y nombre de las organizaciones en las que laboran actualmente nuestros egresados es muy diverso, ya que no predomina alguna actividad o giro en específico. Los tipos de empresas de acuerdo con su actividad o giro son: alimentos, automotriz, bancario, comercial, construcción, educación, entretenimiento, financiero, mobiliaria, logística, recursos humanos, salud, medios digitales y servicios profesionales, entre otros.

$\mathrm{El}_{39.2 \%}$ de los egresados encuestados radica en Puebla, desempeñándose en desiguales cargos y actividades como: analista, asistente ejecutivo, especialista en formación, editor, auxiliar de nóminas, coordinador de talento, coordinador de difusión y conexión universitaria, gerente de proyectos, encargada del área de desarrollo organizacional, ventas, encargado de comunicación social, analista de nómina, coordinador de posgrado, diseñador, analista de $\mathrm{RH}$, community manager, coordinador de reclutamiento y selección, especialista en $\mathrm{RH}$, especialista en reclutamiento y selección de personal, jefe de comunicación y difusión, auxiliar de recursos humanos, Business advisor, asesor de ventas y a servicio, concierge y auxiliar administrativo.

Las actividades principales que desempeñan en sus espacios laborales son: atención a clientes y proveedores, ventas, programación de cursos, seguimiento de capacitación y formación dual para colaboradores, realización de nóminas, contratación de personal, reclutamiento, selección, vinculación universitaria, estrategias de DEO, ruedas de prensa, organización de eventos, creación de boletines informativos, diseños de cursos, planeaciones y propuestas de mejora, redacción de artículos para revista, comunicación interna, estrategias para la planeación y retención del personal, creación de protocolos de comunicación, manejo de la imagen corporativa, diseño de publicidad y comunicación externa.

\section{CONCLUSIONES}

Con los resultados de esta investigación, es importante poner en cuestión el tema que los egresados tienen una percepción de que el mercado laboral es difícil; aunque los encuestados actualmente cuentan con un trabajo en áreas diversas en las que no precisamente es comunicación organizacional.

Ante las respuestas de los egresados sobre la empleabilidad, se denota que estos tienen una empleabilidad positiva al poseer las competencias que exige el mercado laboral, siendo desde el punto de vista de Rentería y Malvezzi (2008) esenciales para desempeñarse en él.

Serrano asevera que el profesional de la comunicación organizacional no puede pretender solamente permanecer con los conocimientos iniciales, esos que obtuvo el estudiante 
en universidad, sino que deberá empaparse del entorno, de diversidad de temas que no necesariamente son de comunicación, sino de todas las disciplinas donde un comunicador tenga entrada a laborar, puede ser desde el campo de la salud, la ingeniería, el gobierno, las ventas, entre otras.

A partir de la presente investigación, se pudo llegar a las siguientes conclusiones como respuesta al objetivo del presente estudio. Una de las principales problemáticas que se presentan en los estudios de egresados está relacionada con la ubicación de los mismos y su disposición hacia la investigación, lo que aumenta su complejidad y restringe la aleatoriedad.

Claramente los resultados de las encuestas reflejan que los espacios laborales en los que se desarrollan los egresados están no solo en la ciudad de Puebla sino en otros estados de la República como: Oaxaca, Veracruz, Tlaxcala, Ciudad de México, Quintana Roo y Morelos y en otros países como Alaska y Estados Unidos de Norteamérica (Santa Ana, California). Además, los 45 egresados que respondieron están trabajando actualmente en medianas y grandes organizaciones públicas y privadas como el Instituto Electoral de la Ciudad de México, Procasa de Orizaba, Audi México, Digital Influence México, SIPS, EY Ernst \& Young, Universidad de las Américas Puebla, G-Logística, Physol, BlueIcon, CONCYTEP, Grupo Carso, Universidad Regional del Sureste Oaxaca, Grupo Turístico La Tlaxcalteca, Pepsico. Mercadotecnia Especializada Kampai, Volkswagen de México, PysesA, Palace Bingo \& Sport Bets Casino, American Express, HSBC MÉXICO S.A, The shore at 46 y Floramundo.

En dichas organizaciones destacan las actividades relacionadas con temas de Recursos Humanos, estrategias de comunicación interna, análisis de contenido, manejo de medios digitales, acciones de comunicación administrativa, manejo de liderazgo, promoción y mercadotecnia (ventas), dirección y gerencia, desarrollo organizacional, comunicación social y diseño. De los giros de las organizaciones donde laboran actualmente destaca el educativo y el automotriz.

De acuerdo con los resultados y dadas las principales actividades de los egresados, se refuerza la necesidad de que el programa académico necesita seguir aplicando a través de las asignaturas de la disciplina en estudio los proyectos de intervención, donde los estudiantes tienen un acercamiento con la realidad, por medio de planes estratégicos y acciones concretas según las necesidades de cada organización. Así mismo, se destaca el trabajo de los egresados fomentando la comunicación integral y el impulso de un estilo de liderazgo transformacional.

Los egresados realizan acciones que impactan en sus stakebolders y desarrollan procesos organizacionales que se desenvuelven en ámbitos de competitividad económica y 
diversidad cultural; sin embargo, no se observa una clara tendencia a la solución de problemas entre la organización y su medio, esto con el fin de conocer y apoyar la estrategia del negocio.

Como parte de las conclusiones también se puede mencionar que el egresado de la disciplina en comunicación organizacional requiere un pensamiento con prospectiva y que esté interesado en los cambios globales, en los avances tecnológicos, pero sobre todo en el recurso humano y las necesidades personales y laborales que a ellos les atañen. Sin olvidar que la base de la comunicación organizacional radica en la estructura y la intención de los mensajes, así como de su capacidad para convocar a todos sus integrantes y grupos de interés.

Claramente se refleja que los egresados de las generaciones en estudio no realizan acciones que tengan una intención o impacto directo en la responsabilidad social organizacional.

Una de las principales problemáticas que se presentan en los estudios de egresados está relacionada con la ubicación de los mismos y su disposición hacia la investigación, lo que aumenta su complejidad y restringe la aleatoriedad.

Sumando al párrafo anterior, y como parte de las conclusiones, se puede percibir que acciones referentes a filosofía organizacional, interculturalidad, ambiente organizacional, clima organizacional, relaciones públicas, comunicación productiva, reputación corporativa, manejo de crisis, fomento de las mejores relaciones cara a cara y entre subsistemas, responsabilidad social organizacional y ética, son conceptos que estas generaciones no llevan a la práctica en sus espacios laborales, esto de acuerdo con los resultados obtenidos. Dicha situación puede ser área de oportunidad para el programa académico en cuanto al desarrollo e impulso de estos conceptos que son parte de la disciplina en comunicación organizacional, que como es sabido estos temas no pueden quedar en nivel teórico sino también práctico, para que los estudiantes en el momento de convertirse en egresados puedan realizar y difundir mensajes que den respuesta a los problemas y necesidades reales de las organizaciones que están sometidas a sociedades complicadas y cambiantes.

Finalmente hay que considerar la necesidad de que los egresados sean proactivos, creativos y disciplinados con sus actividades propias de la línea terminal, con una actitud positiva y abierta a nuevas áreas de conocimiento, así como de una actualización constante y obligada a la multidisciplinariedad, ya que los tiempos actuales exigen una comunicación global y capaz de tener entrada en cualquier espacio laboral. 


\section{REFERENCIAS}

ANUiES, A.E.-L. (3 de marzo de 20I9). Ciclo escolar 20I7-20I8. Recuperado de http://www.anuies. $\mathrm{mx} /$ iinformacion-y-servicios/informacion-estadistica-de-educacion-superior/anuario-estadistico-de-educacion-superior

Bourdieu, P. (2003). Cuestiones de Sociología. Madrid: ISTMO.

Chong, B. (20I6). Introducción. En B. Chong (Coord.) XXIII Anuario de investigación de las Ciencias de la Comunicación (CONEICC).

Díaz, B. F. (1999). Elaboración del perfil profesional. En Metodología de Diseño Curricular para Educación Superior (pp. 85-104). México: Trillas.

Díaz Barriga Arceo, F., \& Hernández Rojas, G. (1999). Estrategias de enseñanza para la promoción de aprendizajes significativos, en estrategias docentes. México: McGraw-Hill.

Federal, G. (3 de marzo de 20I9). Observatorio Laboral Mexicano. Recuperado de https://www.observatoriolaboral.gob.mx/\#/

Fuentes Navarro, R. (I9 de enero de 20I2). Diálogos de la comunicación. Recuperado de http://www. dialogosfelafacs.net/wp-content/uploads/2012/

Guzmán Paz, V. (2012). Comunicación Organizacional. Estado de México: Red Tercer Milenio.

León Duarte, G. A. (2011). Estudios de la Comunicación. Estrategias Metodológicas y Competencias Profesionales en Comunicación. Ciudad de México: Pearson.

Pérez, C. E. (Enero de 20I8). La Ética y la Responsabilidad Social Organizacional, conceptos fundamentales en la formación y desarrollo profesionalde los especialistas de la Comunicación Organizacional en México y España. La Ética y la Responsabilidad Social Organizacional, conceptos fundamentales en la formación y desarrollo profesionalde los especialistas de la Comunicación Organizacional en México y España. Ciudad de México, México.

Puebla, U. P. (I8 de junio de 2000). Rediseño curricular, Licenciatura en Comunicación. Rediseño curricular, argumentación. Puebla, Puebla, México.

Puebla, U. P. (Diciembre 20I7). Rediseño curricular, Licenciatura en Comunicación y Medios Digitales. Rediseño curricular, argumentación. Puebla, Puebla, México.

Queris Rojas, M., Almirall Cabrera, A., Capote García, L., \& Alfonso Robayna, D. (20I2). Diagnóstico del proceso de Comunicación Organizacional. Ingeniería Industrial, I6I-I74.

Rebeil, M. (20I6). Entrevista Desarrollo del Grupo de Investigación 8. Comunicación Integral de las Organizaciones, México. 
Sánchez, O. C. (20I4). Los egresados de comunicación y el mercado laboral: un estudio de trayectorias profesionales. Scielo, $40-45$.

Secretaría de Desarrollo Social. (2011). Cuenta de la Hacienda Pública Federal. Recuperado de http:// www.corresponsabilidad.gob.mx/sistema/sirfosc/seccionpagina/contenido/seccioncontenido/Anexos/archivos/Anexoio7.pdf

Superior, S. D. (4 de marzo de 2019). Reconocimientos de validez oficial de estudios del tipo superior. Recuperado de https://www.sirvoes.sep.gob.mx/sirvoes/mvc/consultas 